\title{
In vitro selection of active hairpin ribozymes by sequential RNA-catalyzed cleavage and ligation reactions
}

\author{
Alfredo Berzal-Herranz, Simpson Joseph, and John M. Burke ${ }^{1}$ \\ Department of Microbiology and Molecular Genetics, Markey Center for Molecular Genetics, The University of Vermont, \\ Burlington, Vermont 05405 USA
}

In vitro selection methods provide rapid and extremely powerful tools for elucidating interactions within and between macromolecules. Here, we describe the development of an in vitro selection procedure that permits the rapid isolation and evaluation of functional hairpin ribozymes from a complex pool of sequence variants containing an extremely low frequency of catalytically proficient molecules. We have used this method to analyze the sequence requirements of two regions of the ribozyme-substrate complex: a 7-nucleotide internal loop within the ribozyme that is essential for catalytic function and substrate sequences surrounding the cleavage-ligation site. Results indicate that only 3 of the 16,384 internal loop variants examined have high cleavage and ligation activity and that the ribozyme has a strong requirement for guanosine immediately 3 ' to the cleavage-ligation site.

[Key Words: Ribozyme; in vitro selection; RNA structure; catalysis]

Received September 23, 1991; accepted November 4, 1991.

The hairpin ribozyme catalyzes a site-specific RNA cleavage reaction that yields products with $5^{\prime}$-hydroxyl and $2^{\prime}, 3^{\prime}$-cyclic phosphate termini (Feldstein et al. 1989; Hampel and Tritz 1989; Haseloff and Gerlach 1989|. In the context of its naturally occurring form, the minus strand of the satellite RNA of tobacco ringspot virus, the reaction is reversible and ligation serves to join the ends of linear monomers to form circular monomeric RNAs (Prody et al. 1986; Van Tol et al. 1991). Figure 1 shows a model of the secondary structure of the complex formed between the hairpin ribozyme and its substrate. This model is based on RNA folding programs and limited phylogenetic and mutational data (Hampel and Tritz 1989; Hampel et al. 1990; Rubino et al. 1990). The ribozyme interacts with its substrate through two short intermolecular helices, one of $6 \mathrm{bp}$ (helix 1) and one of 4 bp (helix 2). Four bases surrounding the cleavage-ligation site (A $\downarrow$ GUC) are drawn as unpaired in Figure 1 but may participate in as yet undetermined interactions, for example, tertiary interactions or a pseudoknot.

Little is known about the structure-function relationship of the hairpin ribozyme. Biochemical methods for identifying important sequences and structures involve site-directed or regional mutagenesis followed by sequencing and activity assays of individual molecular clones. These methods are powerful, but their application is time-consuming and costly. In vivo screening or

${ }^{1}$ Corresponding author. selection schemes with natural or surrogate phenotypes (Price and Cech 1985; Waring et al. 1985) are useful but are difficult to apply to some ribozyme systems, including the system studied here. A further problem is that only three naturally occurring hairpin ribozymes are known (Rubino et al. 1990). They show little sequence variation, so that comparative sequence analysis (James et al. 1989) yields little information concerning their structure and function.

In this paper we describe an in vitro selection method that has been developed in our laboratory to overcome these disadvantages. This method allows either active or inactive ribozymes to be isolated from a large and complex pool of variants. We chose two regions of the complex, one within the ribozyme and one in the substrate, for the initial application of this technique (Fig. 1). The ribozyme target was chosen for initial analysis because it is within an internal loop and is partially protected from nuclease digestion by a noncleavable substrate analog (B. Chowrira and J. Burke, unpubl.|, suggesting that this region of the ribozyme may interact with the substrate or undergo a conformational change upon substrate binding. Results indicate that this internal loop segment has strict sequence requirements and is of critical importance for catalytic function of the enzyme. The substrate target was composed of the 4 unpaired bases surrounding the cleavage-ligation site, as described above. These experiments allow us to conclude that multiple base substitutions at substrate positions $-1,+2$, and +3 do not eliminate cleavage and ligation activity. In striking con- 


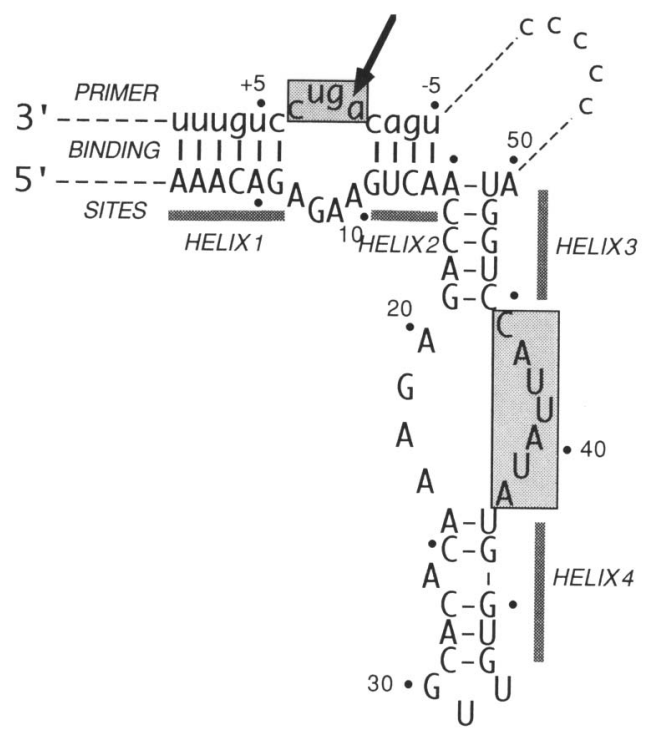

Figure 1. Self-cleaving hairpin ribozyme construct. Secondary structure model of the hairpin ribozyme construct used in this work. Uppercase letters indicate ribozyme; lowercase letters indicate substrate. The arrow indicates cleavage-ligation site. Pentacytidine linker joining ribozyme and substrate is indicated by broken lines. Boxes indicate nucleotides studied in this work. $5^{\prime}$ and $3^{\prime}$ extensions of the sequences serve as primerbinding sites for cDNA synthesis and PCR amplification in the selection experiments.

trast, however, substitutions of $\mathrm{G}_{+1}$, the base immediately to the $3^{\prime}$ side of the cleavage-ligation site, are not tolerated. Recently, we have demonstrated that the 2 -amino group of $G_{+1}$ is an indispensable component of the active site of the hairpin ribozyme (Chowrira et al. 1991).

\section{Results}

In vitro selection

A self-cleaving RNA molecule was constructed for use in the in vitro selection experiments in which the $5^{\prime}$ end of the substrate was tethered to the $3^{\prime}$ end of the ribozyme by a pentacytidine linker (Fig. 1). This molecule was generated by in vitro transcription of a double-stranded synthetic DNA template that was generated from overlapping single-stranded oligonucleotides (Fig. 2). The RNA transcript contains extra sequences at both the $5^{\prime}$ and $3^{\prime}$ ends. These sequences serve as primer-binding sites for cDNA synthesis and amplification during the selection protocol, and they also serve to introduce restriction sites to be used in cloning the amplified cDNA. Sequence variation is introduced into the system during the solid-phase chemical synthesis of DNA by generating base substitutions in the oligonucleotides used to assemble the DNA template for transcription.

The method operates to select molecules that are active for both cleavage and ligation in the following manner (Fig. 2). Active molecules lose the binding site for primer $2(\mathrm{P} 2)$ in the self-cleavage step. After gel purification, the ribozyme-containing $5^{\prime}$-cleavage product, end- ing in a $2^{\prime}, 3^{\prime}$-cyclic phosphate, is incubated with a large molar excess of a mixed RNA-DNA oligomer containing a $5^{\prime} \mathrm{OH}$ that can be ligated to the $3^{\prime}$ ends of the cleavage product in a ribozyme-catalyzed reaction. The $5^{\prime}$ end of this oligomer is RNA and contains nucleotides $G_{+1}$ to $\mathrm{U}_{+9}$ of the substrate (Fig. 1). The $3^{\prime}$ end is DNA and serves as a binding site for a different primer (P3). Thus, molecules active for both cleavage and ligation lose the binding site for P2 and acquire the binding site for P3. Active molecules are selected by initiating cDNA synthesis with P3 and amplifying with primer 1 (P1) and P3, using the polymerase chain reaction (PCR). Inactive molecules are gel purified and then selectively copied into cDNA and amplified using P1 and P2. PCR products are cloned and sequenced to identify variants arising from the positive and negative selections. Activity of molecules selected as active is confirmed by assaying selfcleavage activity of runoff RNAs generated by transcription of linearized plasmids. Sequence analysis of cDNA clones from ribozyme variants selected as inactive serves to confirm that the desired level of sequence variation was introduced into the population of variants. The twostep selection strategy prevents the selection of inactive molecules that have undergone cleavage in trans by active ribozymes. Note that this technique as employed cannot be used to select any variants that are active for self-cleavage but inactive for ligation.

\section{Analysis of a ribozyme internal loop}

Sequences encoding the $3^{\prime}$ segment of the ribozyme in-

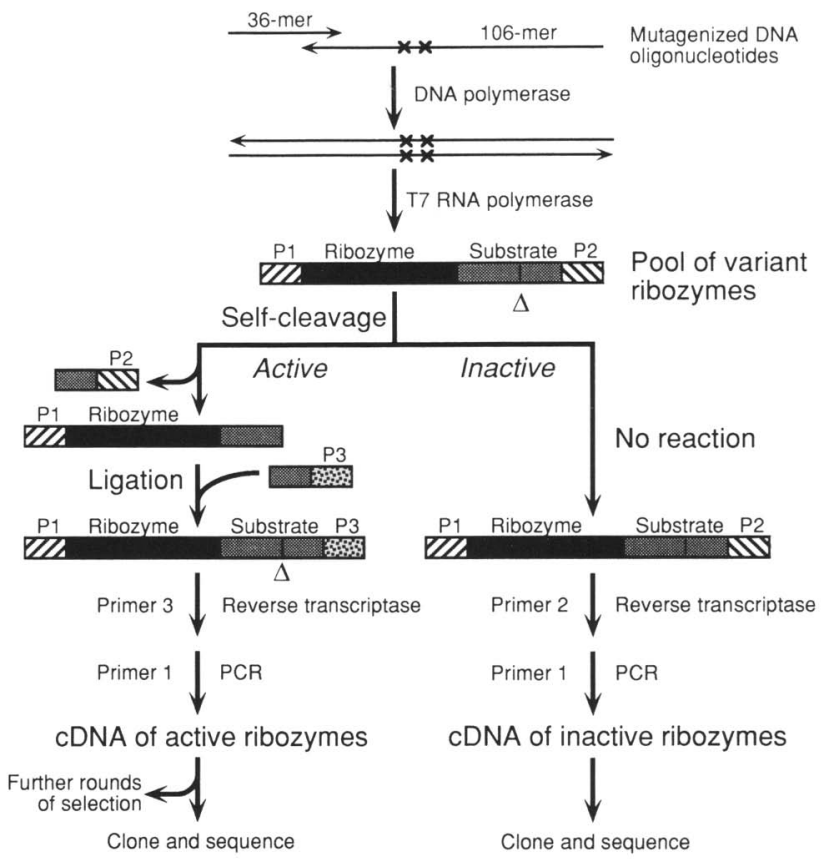

Figure 2. In vitro selection of active and inactive hairpin ribozyme variants. Details of the protocol are given in Materials and methods. $(x)$ Mutation site. (P1-P3) Sequences that serve as primer-binding sites for cDNA synthesis and PCR amplification. 
ternal loop were randomized by incorporating equimolar quantities of $\mathrm{dG}, \mathrm{dA}, \mathrm{dT}$, and $\mathrm{dC}$ into the DNA template at positions corresponding to nucleotides $38-44$ of the ribozyme (Fig. 1), yielding an initial population of $4^{7}$ $(16,384)$ sequence variants. A wild-type template was synthesized as a control. After transcription, the wildtype self-cleaving RNA underwent nearly quantitative cleavage upon synthesis (Fig. 3A, lane 1). In contrast, no cleavage products were observed by autoradiography when the pool of internal loop variants was transcribed (lane 2), indicating that the great majority of internal loop variants are catalytically inactive.

In the ligation reaction, the radiolabeled and gel-purified larger cleavage product, containing the ribozyme and the $5^{\prime}$-substrate segment, was incubated in the presence of a large molar excess of an unlabeled analog of the 3 '-cleavage product (ligation substrate). After optimization of the ligation reaction using the wild-type molecule, the steady-state proportion of ligation product was observed to be $20-30 \%$ of the input ribozyme-containing fragment (Fig. 3B). Efforts to further increase the proportion of ligated reaction products have been unsuccessful, presumably because the product of the ligation reaction can undergo cleavage immediately while it remains bound to the ribozyme.

Using the population of internal loop variants described above, we applied our new method to selectively clone cDNA copies of active and inactive ribozymes (Fig.

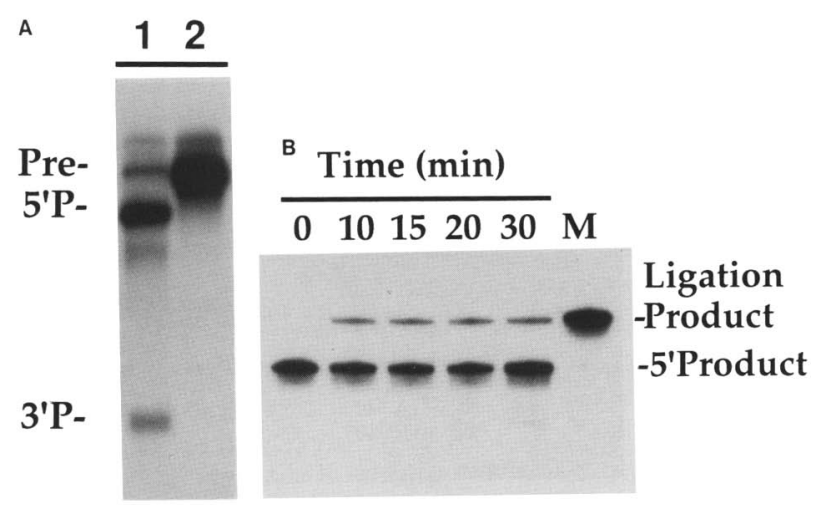

Figure 3. Self-cleavage and ligation by the hairpin ribozyme. (A) Self-cleavage of the RNA obtained by transcription of the wild-type DNA template (lane 1) and the randomized pool of ribozyme internal loop variants (lane 2). (Pre-) Full-length uncut precursor RNA. (5' P-) 5'-Cleavage product of the self-cleaving molecule. This cleavage product contains the ribozyme and substrate sequences to the $5^{\prime}$ side of the cleavage-ligation site. $\left(3^{\prime} \mathrm{P}-\right) 3^{\prime}$-Cleavage product. Assembly and transcription of DNA templates were carried out as described in Materials and methods. An autoradiograph of denaturing $10 \%$ polyacrylamide-urea gel is shown. $(B)$ RNA-catalyzed ligation by the hairpin ribozyme. An amount of 0.1 pmole $(5 \mathrm{nM})$ of the ${ }^{32} \mathrm{P}$-labeled wildtype ribozyme-containing 5 '-cleavage product was incubated with a 600 -fold excess of the unlabeled analog of the 3 '-cleavage product (ligation substrate), as described in Materials and methods for the indicated times. Under these conditions, maximal conversion of $5^{\prime} \mathrm{P}$ to product was $25-30 \%$ and occurred between 20 and $30 \mathrm{~min}$. $(M)$ Purified full-length uncleaved precursor was used as marker.
4). From the library of inactive cDNA clones that were obtained, 20 were chosen randomly for sequencing (Fig. 4A). As expected, sequence variation was only observed in the region corresponding to the internal loop. Among the 20 inactive clones, each of the 4 nucleotides appeared in all seven targeted positions, confirming that the population of molecules was random. Thirty cDNA clones from the cDNA library of active molecules were chosen for sequencing (Fig. 4B). Strikingly, the sequence of each active clone was very close to the wild-type sequence. The wild-type sequence was identified in 11 of the 30 clones. No sequence variation from wild type was observed at four positions of the active molecules, $\mathrm{A}_{38}, \mathrm{U}_{41}$, $\mathrm{U}_{42}$, and $\mathrm{C}_{44}$. Thirteen clones contained only a singlebase substitution at $U_{39}$, changing that base to $C$ (eight clones) or A (five clones). Five clones had two mutations, $\mathrm{U}_{39} \mathrm{C}$ and either $\mathrm{A}_{43} \mathrm{G}$ (four clones) or $\mathrm{A}_{40} \mathrm{C}$ (one clone). One clone had only the $A_{43} G$ substitution.

Self-cleavage assays were carried out to measure the activity of RNA transcripts from the selected cDNAs. Transcripts from each of the five clones chosen randomly from the inactive pool showed no self-cleavage activity (data not shown). All six different sequences selected as active showed significant catalytic activity in vitro (Fig. 4C). Catalytic activity of the six active sequences was proportional to the frequency of the corresponding cDNA clones in the library (see Discussion).

\section{Analysis of the cleavage-ligation site}

For analysis of the 4-base unpaired substrate region surrounding the cleavage-ligation site, we randomized the appropriate sites in the DNA template to generate an initial pool of $4^{4}(256)$ sequence variants. For the selection of active molecules in this experiment, a modification of the procedure was required, as the nucleotides at three of the four mutagenized sites are lost during the first selection step (self-cleavage). Therefore, the second selection step (ligation) used a different ligation substrate in which the 3 bases at the $5^{\prime}$ end were also randomized (positions corresponding to nucleotides +1 , +2 , and +3 ; Fig. 1). Thirty-three clones obtained from the cDNA library of active molecules were sequenced, along with $15 \mathrm{cDNA}$ clones of inactive self-cleaving molecules. Self-cleavage assays indicated that molecules selected as active always showed self-cleavage activity in this assay, except in two cases where additional mutations within the ribozyme (presumably resulting from errors during cDNA synthesis or PCR amplification after cleavage and ligation) were present.

Results of sequencing (Fig. 5) revealed a dramatic difference between the pools of active and inactive molecules. Of the 33 clones of active molecules examined, all contained $G$ at position +1 , whereas none of the inactive molecules contained $G$ at +1 . No significant bias was seen at any of the other positions in either the active or inactive pools. These results demonstrate that all molecules lacking $G_{+1}$ are inactive and strongly suggest that all substrate variants containing $G_{+1}$ have significant self-cleavage and ligation activity. However, it is 


\begin{tabular}{|c|cccccccc|}
$A$ & $A_{38}$ & $U_{39}$ & $A_{40}$ & $U_{41}$ & $U_{42}$ & $A_{43}$ & $C_{44}$ \\
\hline$A$ & 2 & 6 & 3 & 4 & 5 & 4 & 6 \\
C & 8 & 8 & 9 & 7 & 8 & 6 & 8 \\
$G$ & 9 & 2 & 6 & 3 & 3 & 5 & 3 \\
U & 1 & 4 & 2 & 6 & 4 & 5 & 3 \\
\hline
\end{tabular}

\begin{tabular}{|c|cccccccc}
$\mathrm{B}$ & $\mathrm{A}_{38}$ & $\mathrm{U}_{39}$ & $\mathbf{A}_{40}$ & $\mathbf{U}_{41}$ & $\mathbf{U}_{42}$ & $\mathrm{~A}_{43}$ & $\mathrm{C}_{44}$ & Clones \\
\hline 1 & $\cdot$ & $\cdot$ & $\cdot$ & $\cdot$ & $\cdot$ & $\cdot$ & $\cdot$ & 11 \\
2 & $\cdot$ & $\mathrm{A}$ & $\cdot$ & $\cdot$ & $\cdot$ & $\cdot$ & $\cdot$ & 5 \\
3 & $\cdot$ & $\mathrm{C}$ & $\cdot$ & $\cdot$ & $\cdot$ & $\cdot$ & $\cdot$ & 5 \\
4 & $\cdot$ & $\mathrm{C}$ & $\cdot$ & $\cdot$ & $\cdot$ & $\cdot$ & $\cdot$ & 8 \\
5 & $\cdot$ & $\mathrm{C}$ & $\mathrm{C}$ & $\cdot$ & $\cdot$ & $\mathrm{G}$ & $\cdot$ & $\cdot$ \\
6 & $\cdot$ & $\cdot$ & $\cdot$ & $\cdot$ & 1 \\
6 & $\cdot$ & $\cdot$ & $\cdot$ & $\cdot$ & $\cdot$ & $\mathrm{G}$ & $\cdot$ & 1
\end{tabular}

Figure 4. Selection of active and inactive molecules from a randomized pool of ribozyme internal loop variants. $(A)$ Base frequencies of inactive molecules. Twenty cDNA clones of inactive molecules were sequenced. The frequency of nucleotides at each position of the $3^{\prime}$ segment of the ribozyme internal loop (positions 38-44, Fig. 1) is indicated. (B) Clone frequencies of active molecules. Thirty cDNA clones of active molecules were sequenced. Bases differing from wild type are indicated; dots indicate same sequence as wild type. Wild-type sequence is shown at top. Numbers of clones isolated with the indicated sequence are shown at right. $(C)$ Self-cleavage assays, performed as described in Materials and methods. Lane numbers correspond to sequences shown in $B$. Autoradiograph of denaturing $10 \%$ polyacrylamideurea gel is shown.

clear that the sequences at other positions can substantially affect the rate of cleavage, as significant differences in self-cleavage activity were noted among the variant molecules selected as active (data not shown).

\section{Discussion}

In vitro selection techniques (for review, see Abelson

A Active
\begin{tabular}{|ll|ll|}
\hline$A^{\downarrow}$ GGG & $C^{\downarrow}$ GUA & \multicolumn{2}{c|}{ Inactive } \\
$A^{\downarrow}$ GGU & $C^{\downarrow}$ GUG & $C^{\downarrow}$ AAC & $U^{\downarrow}$ AAU \\
$A^{\downarrow}$ GUG & $C^{\downarrow}$ GUU (6) & $C^{\downarrow}$ ACG & $U^{\downarrow}$ CGA \\
$A^{\downarrow}$ GUU & $G^{\downarrow}$ GGC & $C^{\downarrow}$ CAC & $U^{\downarrow}$ UCU \\
$C^{\downarrow}$ GAA (2) & $G^{\downarrow}$ GGG (2) & $C^{\downarrow}$ CAU & \\
$C^{\downarrow}$ GAC (3) & $G^{\downarrow}$ GGU & $C^{\downarrow}$ CCC & \\
$C^{\downarrow}$ GAG (2) & $U^{\downarrow}$ GAC & $C^{\downarrow}$ CCU & \\
$C^{\downarrow}$ GAU (2) & $U^{\downarrow}$ GGU & $C^{\downarrow}$ CUG (2) \\
$C^{\downarrow}$ GCU & $U^{\downarrow}$ GUG & $C^{\downarrow}$ UGG \\
$C^{\downarrow}$ GGA & $U^{\downarrow}$ GUU & $G^{\downarrow}$ AUA \\
$C^{\downarrow}$ GGU (2) & & \\
\hline
\end{tabular}

\begin{tabular}{|c|c|c|c|c|c|}
\hline & $A_{-1}$ & $\mathrm{G}_{+1}$ & $\mathrm{U}_{+2}$ & $\mathrm{C}_{+}$ & Position \\
\hline A & 4 & 0 & 10 & 4 & \\
\hline G & 4 & 33 & 10 & 8 & Active \\
\hline U & 4 & 0 & 12 & 16 & \\
\hline C & 21 & 0 & 1 & 5 & \\
\hline A & 1 & 6 & 4 & 3 & \\
\hline G & 1 & 0 & 2 & 4 & Inactive \\
\hline U & 4 & 2 & 3 & 4 & \\
\hline C & 9 & 7 & 6 & 4 & \\
\hline
\end{tabular}

Figure 5. Selection of active and inactive molecules from a randomized pool of cleavage-ligation site variants. $(A)$ Sequences of active and inactive molecules (clone frequencies). The sequences of 33 active and 15 inactive cleavage-ligation site variants, selected as described in Materials and methods, are shown. The vertical arrow indicates cleavage-ligation site. Boldface letters indicate position +1 . $(B)$ Frequency of occurrence of bases at each position in active and inactive populations.
1990) have recently been applied to the analysis of interactions between RNA and natural or synthetic ligands (Ellington and Szostak 1990; Tuerk and Gold 1990) and the structure-activity relationships of self-splicing group I ribozymes (Green et al. 1990; Robertson and Joyce 1990). These techniques permit the investigator to survey rapidly large numbers of molecules containing multiple base substitutions, without requiring synthesis and characterization of individual variant sequences. In this paper we have described the development of an in vitro selection method for the analysis of the hairpin ribozyme. This method is novel in two respects. First, it is used to analyze a small, trans-acting ribozyme that may be useful for RNA-targeting experiments (Cech 1988); second, it uses a two-step selection based on sequential RNA-catalyzed cleavage and ligation reactions.

The critical feature of the selection of active hairpin ribozymes is the acquisition of a new primer binding site (P3) in the RNA-catalyzed ligation reaction. We assume that ligation proceeds by simple reversal of the cleavage reaction, but this is not firmly established. We expect that an analogous selection method could be developed for other small trans-acting ribozymes, for example, the hammerhead ribozyme, if conditions favoring a ligation reaction can be identified.

We have applied the selection method to two important regions of the ribozyme-substrate complex, one being an internal loop residing entirely within the ribozyme, and the other spanning the cleavage-ligation site of the substrate.

Results of the ribozyme internal loop selection indicate that $A_{38}, U_{41}, U_{42}$, and $C_{44}$ are essential for cleavage-ligation activity of the ribozyme and imply that no base substitutions at these sites may be tolerated under the conditions used for selection. Two models would explain these observations. First, each of these 4 bases could participate in essential secondary or, more likely, tertiary interactions with other sites in the ribozyme. 
Second, one or more of the bases could participate directly in active site chemistry. If tertiary interactions involving these sites are essential, then some [e.g., triple base pairs (Michel et al. 1990)] may be identified with appropriate in vitro selection experiments using variations of the selection method described here. Such experiments are under way in our laboratory. Our results are consistent with the limited phylogenetic data available for this group of ribozymes, which indicates that very little sequence variability is present in this internal loop among the naturally occurring variants (Rubino et al. 1990).

It is significant that each of the four active ribozyme sequences obtained from this selection that is represented by multiple clones (wild type, $\mathrm{U}_{39} \mathrm{~A}, \mathrm{U}_{39} \mathrm{C}$, and $\mathrm{U}_{39} \mathrm{C}: \mathrm{A}_{43} \mathrm{G}$ ) has considerably greater self-cleavage activity than each of the sequences represented by single clones $\left(\mathrm{A}_{43} \mathrm{G}\right.$ and $\left.\mathrm{U}_{39} \mathrm{C}: \mathrm{A}_{40} \mathrm{C}\right)$ (Fig. $\left.4 \mathrm{~B}, \mathrm{C}\right)$. This indicates that the frequency of active variants in the population of cDNA clones is related to the activity of the corresponding variant ribozymes. Thus, subjecting the pool of variants to multiple rounds of selection (Fig. 2) is expected to increase the frequency of the most active ribozymes in the population, allowing the technique to be used to identify ribozymes with optimal cleavage-ligation activity. In the case of a pool of variants containing a very small number of active molecules, such as the ribozyme internal loop randomization described here, multiple rounds of selection are not needed for the identification of optimally active variants.

We expect that it will be necessary to adjust empirically selection conditions or the number of rounds of selection, or both, for each selection experiment that is initiated to obtain the optimal degree of selectivity. Too stringent a selection may result in the loss of informative sequences with slightly suboptimal activity. In contrast, a selection that is not stringent enough will result in a population of variants that is too complex to be characterized adequately by sequencing and activity assays.

The in vitro selection of active molecules after randomization of the bases surrounding the cleavage-ligation site (positions -1 through +3 ) represents a substantially different experiment. In the selection for active molecules, the base at position -1 is subjected to both selective steps. In contrast, the bases at positions +1 through +3 are not subject to selection during the cleavage step, as they are dissociated from the ribozyme- $5^{\prime}$ substrate fragment by the cleavage reaction and are therefore subject to selection only during the ligation step. The observation that there is a strong bias at position +1 in the molecules selected as active directly demonstrates that the ligation step is selective.

Results from the substrate selection experiments indicate that active molecules (selected by ligation) always contain $G$ at the position immediately $3^{\prime}$ of the cleavage-ligation site (position +1 ) and that all molecules lacking $G_{+1}$ are inactive for cleavage and ligation. These results support independent experiments in our laboratory, which have shown that $G_{+1}$ is required for the trans-cleavage reaction, because its 2 -amino group plays an essential role in active site chemistry (Chowrira et al. 1991). Our results strongly suggest that the ribozyme is capable of cleaving all variants at positions $-1,+2$, and +3 , as long as $G_{+1}$, helix 1 , and helix 2 are unchanged. We have documented both self-cleavage and ligation activities for a number of multiple variants, including examples with 3-base substitutions in the internal loop encompassing the cleavage-ligation site. The lack of cleavage of $G_{+1}$ variants that contain $G$ at -1 or +2 demonstrates that there is a strong positional effect for the scissile phosphodiester bond. The structural basis for this positional effect is not known. The common guanosine requirement for both cleavage and ligation supports the notion that ligation is a simple reversal of the cleavage reaction.

Currently, we are using the in vitro selection method to carry out a comprehensive structure-function analysis of the entire ribozyme and substrate. Important applications of the selection system will be, first, to map secondary and tertiary interactions that will be critical in developing an accurate three-dimensional structure model of the ribozyme, and second, to select ribozymes with optimal cleavage activity against foreign target sequences for use as site-specific RNA cleavage tools in vitro, or as mRNA-inactivating reagents in vivo. Furthermore, as the selection scheme is entirely an in vitro process, it can be applied to select variant ribozymes with increased activity under different conditions. For example, if a nuclease-resistant ribozyme is needed for use in the mammalian cytoplasm, then the selection experiments can be carried out in a cytoplasmic extract.

\section{Materials and methods}

Plasmid and bacterial strains

Plasmid pGEM-3Zf $\mid$ - | (Promega) was used for cloning of cDNA from both active and inactive molecules. The Escherichia coli strain DH5 $\alpha(\mathrm{F}$, endA l, hsdR17, supE44, thy-1, recAl, gyrA96,

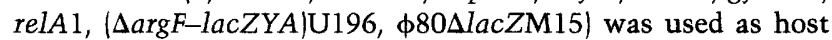
for the individual clones.

\section{Synthesis of oligonucleotides}

All oligonucleotides were synthesized using solid-phase phosphoramidite chemistry on an Applied Biosystems 392 DNARNA synthesizer and purified as described (Scaringe et al. 1990; Chowrira and Burke 1991). Randomization of oligonucleotides was introduced during their synthesis by incorporating equimolar quantities of $\mathrm{dA}, \mathrm{dC}, \mathrm{dG}$, and $\mathrm{dT}$ at targeted positions.

\section{Isolation of active and inactive self-cleaving RNA molecules}

The template for transcription of variant ribozyme pools is assembled by annealing and extension of two overlapping synthetic oligonucleotides, a 36-mer containing a T7 promoter and primer-binding site Pl (5'- TAATACGACTCACTATAGGGTACGCTCCGAATTCGG-3'), and a 106-mer containing antisense sequences of the ribozyme, $C_{5}$ linker, substrate, and primer-binding site P2 (5'-GCGACTGCAGCTCGAGGCAAACAGGACTGTCAGGGGGTACCAGGTAATATACCACAACGTGTGTTTCTCTGGTTGACTTCTCTGTTTCCGAATTCGGAGCGTACCC-3'). Transcription of templates with T7 
RNA polymerase was carried out in a buffer containing 13 $\mathrm{mM} \mathrm{MgCl}_{2}, 2 \mathrm{~mm}$ spermidine, $40 \mathrm{~mm}$ Tris- $\mathrm{HCl}$ ( $\left.\mathrm{pH} 7.5\right)$, and $0.5 \mathrm{mM}$ GTP, ATP, UTP, and CTP. Self-cleavage proceeded in the transcription buffer during the $5-\mathrm{hr}$ incubation at $37^{\circ} \mathrm{C}$. The ribozyme-containing 5 '-cleavage product of the active molecules and uncleaved RNA molecules were gel purified separately.

\section{RNA-catalyzed ligation}

For ligation experiments, the cleavage product containing the ribozyme and the $5^{\prime}$-substrate fragment /terminating with a $2^{\prime}, 3^{\prime}$-cyclic phosphate) was incubated with a large excess 160 100 pmoles) of a ligation substrate (an analog of $\left.3^{\prime} \mathrm{P}\right)$ at $4^{\circ} \mathrm{C}$ in ligation buffer for $30 \mathrm{~min}$ (Fig. 3). The ligation buffer was identical to transcription-cleavage buffer described above, except that $\mathrm{MgCl}_{2}$ was $12 \mathrm{~mm}$ and NTPs were omitted. The ligation substrate was a mixed RNA-DNA polymer containing the sequence corresponding to the $3^{\prime}$-cleavage product and a binding site for P3 (5'-GUCCUGUUUCGCAGATCTAGACGC-3', ribonucleotides underlined, deoxyribonucleotides in plain type). Before the reaction, RNAs were renatured by heating to $95^{\circ} \mathrm{C}$ for $1.5 \mathrm{~min}$ in the reaction buffer, then cooled on ice for $30 \mathrm{~min}$. Reactions were terminated by addition of an equal volume of loading buffer $192 \%$ formamide, $17 \mathrm{~mm}$ EDTA, $0.025 \%$ bromphenol blue, and $0.025 \%$ xylene cyanol). The ligation product was gel purified.

\section{Amplification and sequencing of active and inactive molecules}

cDNA synthesis was initiated by annealing P2 $\left(5^{\prime}\right.$-GCGACTGCAGCTCGAGGC to gel-purified inactive molecules and P3 (GCGTCTAGAGATCTGCG-3') to gel-purified ligation products. Annealing was performed by heating to $70^{\circ} \mathrm{C}$ for $5 \mathrm{~min}$, followed by slow cooling to $40^{\circ} \mathrm{C}$ in $0.4 \times$ reaction buffer. cDNA was synthesized at $40^{\circ} \mathrm{C}$ in a buffer containing $60 \mathrm{mM} \mathrm{NaCl}, 6$ $\mathrm{mM} \mathrm{MgCl}_{2}, 50 \mathrm{~mm}$ Tris- $\mathrm{HCl}$ (pH 8.3), $10 \mathrm{~mm}$ DTT, $1 \mathrm{~mm}$ each dNTP and 98 units of AMV reverse transcriptase (Molecular Genetics Resources). cDNA was amplified by PCR after adding P1 (5'-GGTACGCTCCGAATTCGG-3'), gel-purified, and cloned into plasmid pGEM-3Zf $\mid$-) (Promega) using specific restriction sites contained in P1 (EcoRI), P2 (PstI), and P3 (XbaI). DNA sequence analysis (Sanger et al. 1977) of small-scale plasmid preparations was done by using standard methods.

\section{Self-cleavage assays}

For the self-cleavage assays, ${ }^{32} \mathrm{P}$-labeled transcripts were generated by runoff transcription of BglII- or XhoI-digested plasmids, for clones selected as active or inactive, respectively. (These restriction enzyme sites are encoded in $\mathrm{P} 3$ or P2, respectively.) Transcription was carried out for $2 \mathrm{hr}$ as described above, except $0.01 \%$ of Triton $\mathrm{X}-100$ was added.

\section{Acknowledgments}

This work was supported by grants from the National Institutes of Health. A.B.-H. was supported by a NATO postdoctoral fellowship.

The publication costs of this article were defrayed in part by payment of page charges. This article must therefore be hereby marked "advertisement" in accordance with 18 USC section 1734 solely to indicate this fact.

\section{References}

Abelson, J. 1990. Directed evolution of nucleic acids by independent replication and selection. Science 249: 488-489.
Cech, T.R. 1988. Ribozymes and their medical implications. IAMA 260: 3030-3034.

Chowrira, B.M. and J.M. Burke. 1991. Binding and cleavage of nucleic acids by the "hairpin" ribozyme. Biochemistry 30: 8518-8522.

Chowrira, B.M., A. Berzal-Herranz, and J.M. Burke. 1991. Novel guanosine requirement for catalysis by the hairpin ribozyme. Nature (in press).

Ellington, A.D. and J.W. Szostak. 1990. In vitro selection of RNA molecules that bind specific ligands. Nature 346: 818822.

Feldstein, P.A., J.M. Buzayan, and G. Bruening. 1989. Two sequences participating in the autolytic processing of satellite tobacco ringspot virus complementary RNA. Gene 82: 5361.

Green, R., A.D. Ellington, and J.W. Szostak. 1990. In vitro genetic analysis of the Tetrahymena self-splicing intron. $\mathrm{Na}$ ture 347: 406-408.

Hampel, A. and R. Tritz. 1989. RNA catalytic properties of the minimum (-) sTRSV sequence. Biochemistry 28: 49294933.

Hampel, A., R. Tritz, M. Hicks, and P. Cruz. 1990. "Hairpin" catalytic RNA model: Evidence for helices and sequence requirement for substrate RNA. Nucleic Acids Res. 18: 299304.

Haseloff, J. and W.L. Gerlach. 1989. Sequences required for selfcatalysed cleavage of the satellite RNA of tobacco ringspot virus. Gene 82: 43-52.

James, B.D., G.J. Olsen, and N.R. Pace. 1989. Phylogenetic comparative analysis of RNA secondary structure. Methods Enzymol. 180: 227-238.

Michel, F., A.D. Ellington, S. Couture, and J.W. Szostak. 1990. Phylogenetic and genetic evidence for base-triples in the catalytic domain of group I introns. Nature 347: 578-580.

Price, J. V. and T.R. Cech. 1985. Coupling of Tetrahymena ribosomal RNA splicing to $\beta$-galactosidase expression in E. coli. Science 228: 719-722.

Prody, G.A., J.T. Bakos, J.M. Buzayan, I.R. Schneider, and G. Bruening. 1986. Autolytic processing of dimeric plant virus satellite RNA. Science 231: 1577-1580.

Robertson, D.L. and G.F. Joyce. 1990. Selection in vitro of an RNA enzyme that specifically cleaves single-stranded DNA. Nature 344: 467-468.

Rubino, L., M.E. Tousignant, G. Steger, and J.M. Kaper. 1990. Nucleotide sequence and structural analysis of two satellite RNAs associated with chicory yellow mottle virus. J. Gen. Virol. 71: 1897-1903.

Sanger, F., S. Nicklen, and A.R. Coulson. 1977. DNA sequencing with chain-terminating inhibitors. Proc. Natl. Acad. Sci. 74: 5463-5467.

Scaringe, S.A., C. Francklyn, and N. Usman. 1990. Chemical synthesis of biologically active oligoribonucleotides using beta-cyanoethyl protected ribonucleoside phosphoramidites. Nucleic Acids Res. 18: 5433-5441.

Tuerk, C. and L. Gold. 1990. Systematic evolution of ligands by exponential enrichment: RNA ligands to bacteriophage T4 DNA polymerase. Science 249: 505-510.

Van Tol, H., J.M. Buzayan, and G. Bruening. 1991. Evidence for spontaneous circle formation in the replication of the satellite RNA of tobacco ringspot virus. Virology 180: 23-30.

Waring, R.B., J.A. Ray, S.W. Edwards, C. Scazzocchio, and R.W. Davies. 1985. The Tetrahymena rRNA intron self-splices in E. coli: In vivo evidence for the importance of key basepaired regions of RNA for RNA enzyme function. Cell 40: $371-380$. 


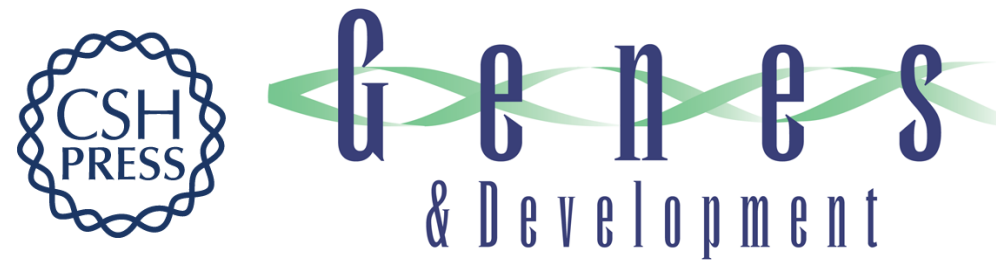

\section{In vitro selection of active hairpin ribozymes by sequential RNA-catalyzed cleavage and ligation reactions.}

A Berzal-Herranz, S Joseph and J M Burke

Genes Dev. 1992, 6:

Access the most recent version at doi:10.1101/gad.6.1.129

References This article cites 20 articles, 5 of which can be accessed free at: http://genesdev.cshlp.org/content/6/1/129.full.html\#ref-list-1

License

Email Alerting

Service

Receive free email alerts when new articles cite this article - sign up in the box at the top right corner of the article or click here.

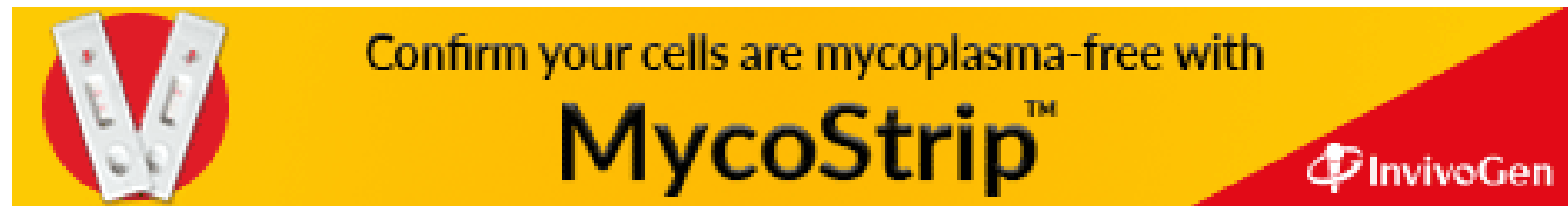

